\title{
Synthesis and Anticancer Activity of Mono-Carbonyl Analogues of Curcumin
}

\author{
Song Yin ${ }^{1,2,3}$, Xing Zheng ${ }^{1,2 *}$ Xu Yao ${ }^{1}$, Yuhong Wang ${ }^{2}$, Duanfang Liao ${ }^{2}$ \\ ${ }^{1}$ Institute of Pharmacy and Pharmacology, University of South China, Hengyang, China; ${ }^{2}$ Division of Stem Cell Regulation and Ap- \\ plication, State Key Laboratory of Chinese Medicine Powder and Medicine Innovation in Hunan (Incubation), Hunan University of \\ Chinese Medicine, Changsha, China; ${ }^{3}$ The Institute of Medicinal Plant Development, Chinese Academy of Medical Sciences, Beijing, \\ China. \\ Email: " pharmacynhu@sohu.com
}

Received November $8^{\text {th }}, 2012$; revised December $10^{\text {th }}, 2012$; accepted December $18^{\text {th }}, 2012$

\begin{abstract}
Curcumin has been reported to possess multifunctional bioactivities with low toxicity. However, the clinical application of curcumin has been significantly limited by its instability and poor metabolic property. Up to now, multiple approaches are being sought to overcome these limitations to obtaining "super curcumin", and many analogues of curcumin have been designed and synthesized. In all of those analogues, a series of mono-carbonyl curcumin analogues deleting the $\beta$-diketone draw our attention. Since the seven-carbon $\beta$-diketone linker in curcumin may be responsible for its instability, the series of mono-carbonyl curcumin analogues deleting the $\beta$-diketone may be potential prodrug with improved pharmacokinetic and pharmacodynamic properties. This review just focuses on these more stable mono-carbonyl analogues of curcumin, and shows the new class of active structure by introducing the synthesis and anticancer activity of them.
\end{abstract}

Keywords: Curcumin; Mono-Carbonyl Analogues; Anticancer Activity; SAR

\section{Introduction}

Curcumin,1,7-bis-(3-hydroxy-4-methoxyphenyl)-1,6-heptadiene-3,5-dione, (Figure 1) is a key active component in the traditional herb Curcuma Longa and has been used for centuries throughout Asia as a food additive, cosmetic, and as a traditional herbal medicine. As a spice, it provides curry with its distinctive color and flavor. Furthermore, traditional Indian medicine has considered curcumin a drug effective for various respiratory conditions (asthma, bronchial hyperactivity, and allergy) as well as for other disorders including anorexia, coryza, cough, hepatic diseases, and sinusitis [1,2]. Over the past decade, several studies have substantiated the potential prophylactic or therapeutic value of curcumin and have unequivocally supported reports of its potential biological benefits, including anti-tumor, anti-inflammation, cardio-protection and anti-virus [3-6]. Clinical studies reported that curcumin could be orally administered up to $12 \mathrm{~g} /$ day without any toxic effect in humans [7]. In spite of the favorable biological properties and low toxicity of curcumin, there are drawbacks that limit the development of curcumin as a potential therapeutic agent, including low bioavailability and instability at neutral to basic con-

${ }^{*}$ Corresponding author. ditions [8,9]. Multiple approaches have been sought to overcome these limitations, and many analogues of have been designed and synthesized [10-16].

Evidences from both in vitro and in vivo studies show that the $\beta$-diketone moiety is responsible for the instability and weak pharmacokinetic profiles of curcumin. In vitro, curcumin is unstable at a $\mathrm{pH}$ above 6.5 because of the highly reactive $\beta$-diketone moiety in the structure of curcumin $[17,18]$. And in vivo, recent studies indicate that the $\beta$-diketone moiety appears to be a specific substrate of a series of aldo-keto reductases [19-21] and can be decomposed rapidly. In 2009, G. Liang et al. [22] have made some studies on in vitro stability and in vivo pharmacokinetic about a series of mono-carbonyl analogues of curcumin, and the results indicated that the stability of these mono-carbonyl analogues was greatly enhanced in vitro and their pharmacokinetic profiles were also significantly improved in vivo. And in some recent<smiles>COc1ccc(/C=C/C(=O)CC(=O)/C=C/c2ccc(OC)c(O)c2)cc1O</smiles>

Figure 1. Structure of curcumin. 
studies,some activities of the mono-carbonyl analogues of curcumin are even better than curcumin. This review will focus on these more stable mono-carbonyl analogues of curcumin.

\section{Anticancer Activity of Curcumin and Its Limitations}

In all of those biological activities of curcumin, the anticancer activity was investigated more. In basic cancer research, Curcumin's beneficial effects have been shown in prostate cancer cells [23], ovarian cancer cells [24], gastric carcinoma cells [25], liver cancer cells, hepatocellular carcinoma cells [26], cervical cancer cells [27], breast cancer cells [28], colon cancer cells [29], lung carcinoma cell [30], bladder cancer cells [31], pancreatic adenocarcinoma cell [32] and so on, providing strong preliminary data for the justification of clinical studies in humans. Probably the first indication of curcumin's anticancer activities in human participants was shown in 1987 by Kuttan and co-workers [33], who conducted a clinicaltrial involving 62 patients with external cancerous lesions. Topical curcumin was found to produce remarkable symptomatic relief as evidenced by reductions in smell, itching, lesion size, and pain. Although the effect continued for several months in many patients, only one patient had an adverse reaction [33]. Since then, curcumin, either alone or in combination with other agents, has demonstrated potential against colorectal cancer [34], pancreatic cancer [35], breast cancer [36], prostate cancer [37], multiple myeloma [38], lung cancer [39], oral cancer [40], and head and neck squamous cell carcinoma (HNSCC) [41]. To date, no studies have reported any toxicity associated with the use of curcumin in either animals or humans.

However studies over the past several decades related to absorption, distribution, metabolism and excretion of curcumin have revealed poor absorption and rapid metabolism of curcumin that severely curtails its bioavailability. For example, Wahlstrom and Blennow in 1978 reported the first study to examine the uptake, distribution, and excretion of curcumin using Sprague-Dawley rats. They found negligible amounts of curcumin in blood plasma of rats after oral administration of $1 \mathrm{~g} / \mathrm{kg}$ curcumin, indicating its poor absorption from the gut [42]. Similarly, in a study by Pan et al., a curcumin dose of 0.1 $\mathrm{g} / \mathrm{kg}$ i.p. produced the maximum amount of curcumin in the intestine $(117 \mu \mathrm{g} / \mathrm{g})$ one hour after dosing. Spleen, liver, and kidney showed moderate curcumin amounts of $26.1,26.9$, and $7.5 \mu \mathrm{g} / \mathrm{g}$, respectively whereas only a trace amount $(0.4 \mu \mathrm{g} / \mathrm{g})$ was found in brain tissue [43].

\section{Synthesis of the Mono-Carbonyl Analogues of Curcumin}

Organic synthesis provides a cheaper and more efficient approach to obtain large amounts of 'natural inspired' chemicals to therapeutics than by extracting few milligrams from the nature. The synthesis of this kind of analogues of curcumin is based on aldol condensation. Many scholars have explored the method to this kind of analogues.

As early as in 1997 , owing to the unstable $\beta$-diketone moiety in curcumin, Sardjiman et al. [44] had synthesized a series of mono-carbonyl analogues of curcumin by coupling the appropriate aromatic aldehyde with cyclohexanone, cyclopentanone or acetone in the presence of concentrated hydrochloric acid. And then Z.-Y. Du et al. [45] synthesized some others mono-carbonyl analogues of curcumin based on the procedure of Sardjiman. A mixture of the appropriate aldehyde (2 equiv) and the ketone (1 equiv) was dissolved in glacial acetic acid saturated with anhydrous hydrogen chloride and heated in a water bath at $25^{\circ} \mathrm{C}-30^{\circ} \mathrm{C}$ for $2 \mathrm{~h}$. After standing for $2 \mathrm{~d}$, the mixture was treated with cold water and filtered. The solid obtained was then washed and dried. The crude product was recrystallized from appropriate solvents (methanol or ethanol). In 2009, K.-H. Lee et al. [46] got some mono-carbonyl analogues of curcumin with better procedure with shorter reaction time. A mixture of the aromatic aldehyde ( 2 equiv) and the appropriate ketone ( 1 equiv) was dissolved in $15 \mathrm{ml}$ of ethanol in a single necked round bottomed flask and stirred for several minutes at $5^{\circ} \mathrm{C}$ (ice bath). Into this solution $10 \mathrm{ml}$ of a $40 \%$ $\mathrm{NaOH}$ solution in water was then added drop wise over several minutes. The mixture is then allowed to stir at room temperature for approximately $10 \mathrm{~h}$. The reaction was neutralized with a dilute $\mathrm{HCl}$ solution to form a precipitate, which was then collected by suction filtration. The product, obtained after removal of the solvent under reduced pressure, was crystallized from an appropriate solvent. G. Liang et al. [47] also got some compounds under alkaline conditions. Instead of ethanol/sodium hydroxide, they got a higher yied in the solution of methanol/sodium methoxide. A method for the preparation of an kind of mono-carbonyl analogues of curcumin, by Claisen condensation, starting from vanillin or vanillin derivatives, respectively, and acetone in acid medium under ultrasonic irradiation conditions with good yield and purity, which was patented in 2002, was adopted by J. A. Quincoces Suarez et al. [48]. They also synthesized some mono-carbonyl analogues of curcumin under ultrasonic irradiation $(40 \mathrm{kHz})$ in the presence of concentrated hydrochloric acid with good yield.

All the reported procedures for the preparation of the mono-carbonyl analogues of curcumin are divided into two conditions, namely under acidic conditions or alkaline conditions. Both of the two procedures could gain a high yied, and that can provided the basis for the further studies of pharmacological activity (Figure 2). 


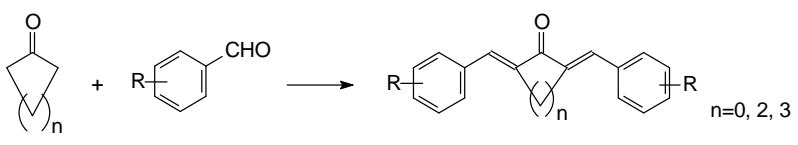

Figure 2. Synthesis of the mono-carbonyl analogues of curcumin. Conditions: $\mathrm{HCl} / \mathrm{EtOH}, \mathrm{NaOH} / \mathrm{CH}_{3} \mathrm{OH}, \mathrm{NaOH}$.

\section{Anticancer Activity of the Mono-Carbonyl Analogues of Curcumin}

It has been reported that curcumin possesses a widespectrum of anti-tumor properties [4,5]. The mono-carbonyl analogues derived from curcumin has also been reported possessing a wide-spectrum of anti-tumor properties. That will be strong basis for further studies about anticancer activity of these mono-carbonyl analogues.

H. Ligeret et al. [49] synthesized two fluoride curcumin derivatives and studied their effects on mitochondrial. They induced the collapse of mitochondrial membrane potential, increased mitochondrial respiration, and decreased $\mathrm{O}^{2-}$ production and promoted $\mathrm{Ca}^{2+}$ release. These effects were reversed by the recoupling agent 6-Ketocholestanol, but not by cyclosporin A, an inhibitor of the permeability transition pore (PTP), suggesting that these compounds act as uncoupling agents. Compound 71 , one of the two fluoride curcumin derivatives, is a mono-carbonyl analogue. It was known to all that treatment with uncouplers can induce apoptotic cell death in tumor lines [50]. Therefore, compound 71 may represent interesting antitumor agent.

B. K. Adams et al. [51] synthesized thirteen monocarbonyl analogues of curcumin and screened for anticancer and antiangiogenesis activities at Emory University and at the National Cancer Institute (NCI). The majority of the analogs demonstrated a moderate degree of anti-cancer activity. And compounds 77 and 98 exhibited a high degree of cytotoxicity, in addition, these compounds inhibit tumor cell growth with a higher potency than the commonly used chemotherapeutic drug, cisplatin. Sun et al. [52] used compound 98 to propose a new drug delivery system, drug-linker-Phe-Phe-Argmk-fVIIa, which can associate with TF on the surface of cancer cells, but release the cytotoxic agent in the cytoplasm, for a further study. The result showed when breast cancer cells (MDA-MB-231) and human melanoma cells (RPMI-7951) are treated with the complex, the cells are arrested to a greater extent than compound 98 alone by comparison with controls. Kasinski et al. [53] demonstrate that compound 11 induces death of lung, breast, ovarian, and cervical cancer cells, with a potency about ten times higher than that of curcumin.

Youssef et al. [54,55] tested the anticancer activity of fifteen mono-carbonyl analogues of curcumin they synthesized. The result revealed that compound 95 shows high anticancer activity.
H. Ohori et al. [56] selected forty-five compounds structurally analogous to curcumin, resveratrol or capsaicin and tested their abilities to suppress the growth of the colon cancer cell line DLD-1. Only one compound, compound 34, which is nominated as an mono-carbonyl analogue of curcumin, was found to have a stronger ability to suppress the growth of DLD-1 compared with curcumin. The growth-suppressive activity of compound 34 was examined in other cancer cell lines, including lines derived from stomach (GCIY, SH10TC), lung (LK87), breast (MCF7), ovary (OVK18), prostate (PC3), pancreas (PK9), bile duct (HuCCT1), thyroid gland (8505c), skin (A431), kidney (ACHN), and liver cancers (HepG 2) and also melanoma (G361), and the compound also showed high growth-suppressive activity. Five related compounds were also synthesized and tested to be having high growth-suppressive activity. In the subsequent studies, H. Shibata et al. [57] examined the availability of compound 6 and its oxicity, which showed a thirtyfold greater growth suppression in vitro via similar molecular mechanisms to curcumin, by using the familial adenomatous polyposis (FAP) mouse, in vivo. In this study, oral administration of compound 6 improved chemopreventive ability in FAP mouse without apparent toxicities in vivo.

H. Chandru et al. [58] synthesized four novel dienone cyclopropoxy curcumin analogs $8-11$ by nucleophilic substitution reaction with cyclopropyl bromide and studied their tumor inhibitory and antiangiogenic effects on mouse Ehrlich ascites tumor (EAT) in vivo. Their findings demonstrated that the tumor growth inhibitory effects of synthetic mono-carbonyl analogues of curcumin $8-11$ could be mediated by promoting apoptosis and inhibiting tumor angiogenesis.

G. Liang et al. [22] designed and synthesized a series of mono-carbonyl analogues of curcumin and examined their stability in vitro and pharmacokinetic in vivo, the results indicated that the stability of these mono-carbonyl analogues was greatly enhanced in vitro and their pharmacokinetic profiles were also significantly improved in vivo. Furthermore, the cytotoxic activities of mono-carbonyl analogues were evaluated in seven different tumor cell lines by MTT assay. The results suggested that the five-carbon linker-containing analogues of curcumin may be favorable for the curcumin-based drug development both pharmacokinetically and pharmacologically.

J. R. Fuchs et al. [59] have done a research for structure-activity relationship of curcumin analogues. And in their studies ten mono-carbonyl analogues of curcumin were synthesized and evaluated. The most potent compound, compound 39, showed potent growth inhibitory activities on both prostate and breast cancer lines with IC50 values in sub-micromolar range, fifty times more potent than curcumin. 
B. Yadav et al. [60] synthesised a series of eighteen heterocyclic cyclohexanone analogues of curcumin and screened for their activity in both adherent and non-adherent cancer cell models. And found some of the compounds showed potent cytotoxicity towards MBA-MB231, MDA-MB-468, SkBr3 cell lines and inhibition of NF-jB activation and were also able to cause MDAMB-231 cells to undergo apoptosis after $18 \mathrm{~h}$. This level of activity warrants further investigation for the treatment of ER-negative breast cancer and/or chronic myelogenous leukemia as prototypical cellular models for solid and liquid tumors.

H. Yamakoshi et al. [61] synthesized a series of monocarbonyl analogues of curcumin and evaluated for their cytotoxicities against human colon cancer cell line HCT116 to conclude the SAR of mono-carbonyl analogues of curcumin for further development of their use in cancer chemotherapy.

P. Lagisetty et al. [62] obtained a potent and novel curcuminoid 161 from structure activity relationship of 3,5-bis(benzylidene)-4-piperidones for their anti-proliferative activity in lung adenocarcinoma $\mathrm{H} 441$ cells.

J. A. Quincoces Suarez et al. [48] found that the set of synthetic compound 21 exhibited high antitumoral activeties regarding in vitro screening against several human tumor cell lines as lung carcinoma NCI-460, melanoma UACC-62, breast MCF-7, colon HT-29, renal 786-O, ovarian OVCAR-03 and ovarian expressing the resistance phenotype for adriamycin NCI-ADR/RES, prostate PC-3, and leukemia K-562.

J. Xiao et al. [63] synthesized a new mono-carbonyl analogue of curcumin 18 and investigate the apoptosis of human hepatocellular carcinoma cell line HepG 2. The result suggests that 18 has more potent antitumor activity than curcumin, which is associated with activation of ER stress and induction of apoptosis in HepG 2 cells.

All of those reported about the anticancer activity of the mono-carbonyl analogues of curcumin indicated that this kind of compounds may provided a series of nice leading compounds with anticancer activity. And all compounds with anticancer activity show in Tables 1-4.

\section{Structure Activity Relationship Analysis of the Mono-Carbonyl Analogues of Curcumin}

As mentioned above, many researchers have studied about anticancer activity of mono-carbonyl analogues of curcumin, SAR about anticancer activity have been also discussed. In the research group of G Liang, they have concluded the SAR of mono-carbonyl analogues from their data: 1) A strong electron-withdrawing substituent in 2'position may increase bioactivity and the more electronegative is the moiety, the more cytotoxic is the compound; 2) A weak electron-donating substituent in 4'position is most favorable to the anti-tumor activity of

Table 1. Compounds 1 - 48.

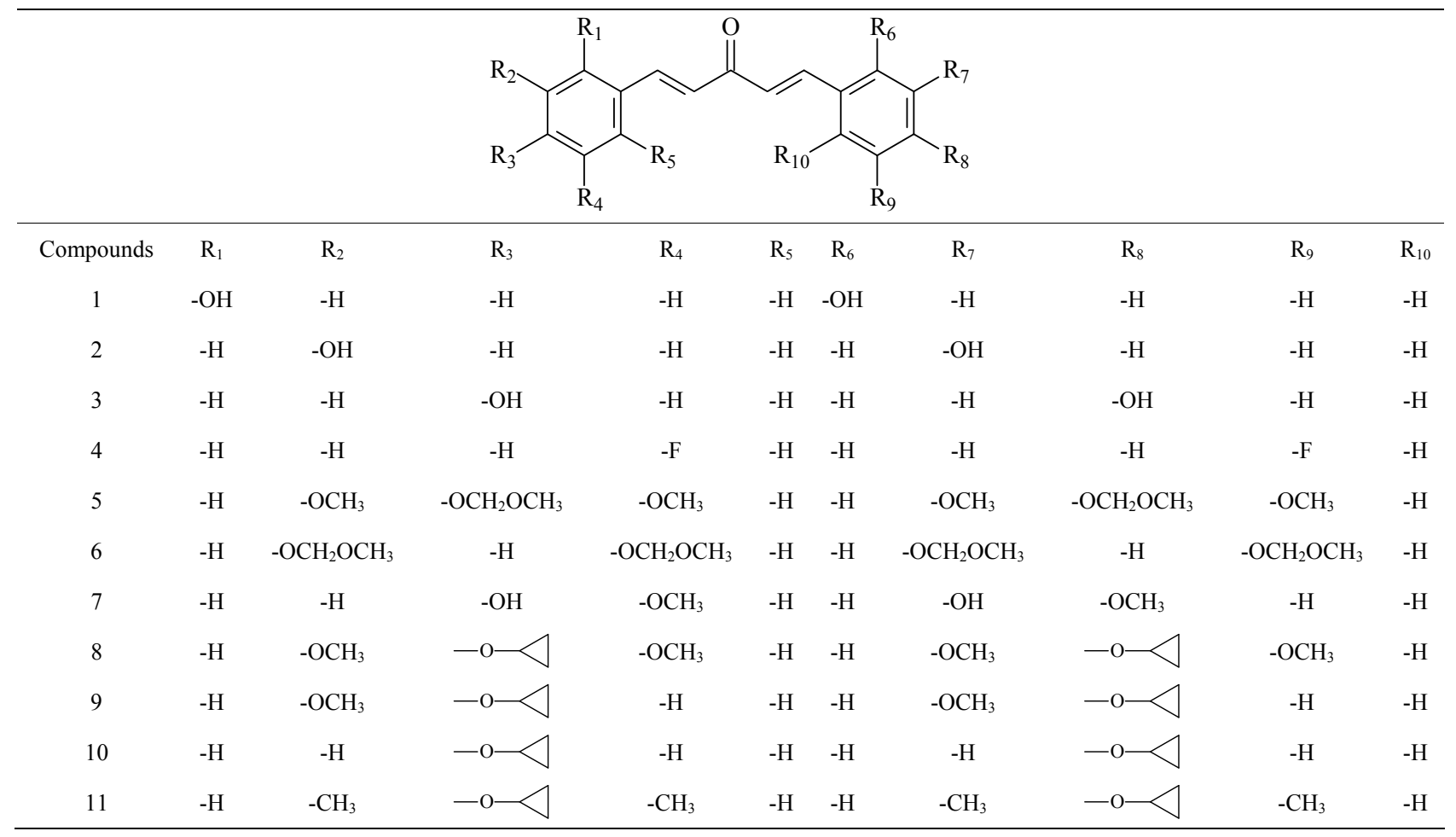




\section{Continued}

\begin{tabular}{|c|c|c|c|c|c|c|c|c|c|c|}
\hline 12 & $-\mathrm{H}$ & $-\mathrm{H}$ & $-\mathrm{OCH}_{3}$ & $-\mathrm{OH}$ & $-\mathrm{H}$ & $-\mathrm{H}$ & $-\mathrm{OCH}$ & $-\mathrm{OH}$ & $-\mathrm{H}$ & $-\mathrm{H}$ \\
\hline 13 & $-\mathrm{H}$ & $-\mathrm{H}$ & $-\mathrm{OCH}_{2} \mathrm{CH}=\mathrm{CH}_{2}$ & $-\mathrm{H}$ & $-\mathrm{H}$ & $-\mathrm{H}$ & $-\mathrm{H}$ & $-\mathrm{OCH} 2 \mathrm{CH}=\mathrm{CH}_{2}$ & $-\mathrm{H}$ & $-\mathrm{H}$ \\
\hline 14 & $-\mathrm{H}$ & $-\mathrm{H}$ & $\begin{array}{c}\text {-(Terahyro-2H- } \\
\text { pyran-yl)oxy }\end{array}$ & $-\mathrm{H}$ & $-\mathrm{H}$ & $-\mathrm{H}$ & $-\mathrm{H}$ & $\begin{array}{c}\text {-(Terahyro-2H- } \\
\text { pyran-yl)oxy }\end{array}$ & $-\mathrm{H}$ & $-\mathrm{H}$ \\
\hline 15 & $-\mathrm{CH}_{3}$ & $-\mathrm{H}$ & $-\mathrm{H}$ & $-\mathrm{CH} 3$ & $-\mathrm{H}$ & $-\mathrm{CH}_{3}$ & $-\mathrm{H}$ & $-\mathrm{H}$ & $-\mathrm{CH}_{3}$ & $-\mathrm{H}$ \\
\hline 16 & $-\mathrm{H}$ & $-\mathrm{H}$ & $-\mathrm{C}\left(\mathrm{CH}_{3}\right)_{3}$ & $-\mathrm{H}$ & $-\mathrm{H}$ & $-\mathrm{H}$ & $-\mathrm{H}$ & $-\mathrm{C}\left(\mathrm{CH}_{3}\right)_{3}$ & $-\mathrm{H}$ & $-\mathrm{H}$ \\
\hline 17 & $-\mathrm{H}$ & $-\mathrm{H}$ & $-F$ & $-\mathrm{H}$ & $-\mathrm{H}$ & $-\mathrm{H}$ & $-\mathrm{H}$ & $-F$ & $-\mathrm{H}$ & $-\mathrm{H}$ \\
\hline 18 & $-\mathrm{Br}$ & $-\mathrm{H}$ & $-\mathrm{H}$ & $-\mathrm{H}$ & $-\mathrm{H}$ & $-\mathrm{Br}$ & $-\mathrm{H}$ & $-\mathrm{H}$ & $-\mathrm{H}$ & $-\mathrm{H}$ \\
\hline 19 & $-\mathrm{Cl}$ & $-\mathrm{H}$ & $-\mathrm{H}$ & $-\mathrm{H}$ & $-\mathrm{H}$ & $-\mathrm{Cl}$ & $-\mathrm{H}$ & $-\mathrm{H}$ & $-\mathrm{H}$ & $-\mathrm{H}$ \\
\hline 20 & $-\mathrm{CF}_{3}$ & $-\mathrm{H}$ & $-\mathrm{H}$ & $-\mathrm{H}$ & $-\mathrm{H}$ & $-\mathrm{CF}_{3}$ & $-\mathrm{H}$ & $-\mathrm{H}$ & $-\mathrm{H}$ & $-\mathrm{H}$ \\
\hline 21 & $-\mathrm{H}$ & $-\mathrm{OCH}_{3}$ & $-\mathrm{OH}$ & $-\mathrm{H}$ & $-\mathrm{H}$ & $-\mathrm{H}$ & $-\mathrm{OCH}_{3}$ & $-\mathrm{OH}$ & $-\mathrm{H}$ & $-\mathrm{H}$ \\
\hline 22 & $-\mathrm{H}$ & $-\mathrm{OH}$ & $-\mathrm{OCH}_{3}$ & $-\mathrm{H}$ & $-\mathrm{H}$ & $-\mathrm{H}$ & $-\mathrm{OH}$ & $-\mathrm{OCH}_{3}$ & $-\mathrm{H}$ & $-\mathrm{H}$ \\
\hline 23 & $-\mathrm{H}$ & $-\mathrm{OCH}_{3}$ & $-\mathrm{OH}$ & $-\mathrm{OCH}_{3}$ & $-\mathrm{H}$ & $-\mathrm{H}$ & $-\mathrm{OCH}_{3}$ & $-\mathrm{OH}$ & $-\mathrm{OCH}_{3}$ & $-\mathrm{H}$ \\
\hline 24 & $-\mathrm{H}$ & $-\mathrm{OCH}_{3}$ & $-\mathrm{OSO}_{2} \mathrm{NH}_{2}$ & $-\mathrm{H}$ & $-\mathrm{H}$ & $-\mathrm{H}$ & $-\mathrm{OCH}_{3}$ & $-\mathrm{OSO}_{2} \mathrm{NH}_{2}$ & $-\mathrm{H}$ & $-\mathrm{H}$ \\
\hline 25 & $-\mathrm{H}$ & $-\mathrm{H}$ & $-\mathrm{OSO}_{2} \mathrm{NH}_{2}$ & $-\mathrm{OCH}_{3}$ & $-\mathrm{H}$ & $-\mathrm{H}$ & $-\mathrm{H}$ & $-\mathrm{OSO}_{2} \mathrm{NH}_{2}$ & $-\mathrm{OCH}_{3}$ & $-\mathrm{H}$ \\
\hline 26 & $-\mathrm{H}$ & $-\mathrm{OCH} 3$ & $-\mathrm{OSO}_{2} \mathrm{NH}_{2}$ & $-\mathrm{OCH}_{3}$ & $-\mathrm{H}$ & $-\mathrm{H}$ & $-\mathrm{OCH}_{3}$ & $-\mathrm{OSO}_{2} \mathrm{NH}_{2}$ & $-\mathrm{OCH}_{3}$ & $-\mathrm{H}$ \\
\hline 27 & $-\mathrm{OCH}_{3}$ & $-\mathrm{H}$ & $-\mathrm{H}$ & $-\mathrm{H}$ & $-\mathrm{H}$ & $-\mathrm{OCH}_{3}$ & $-\mathrm{H}$ & $-\mathrm{H}$ & $-\mathrm{H}$ & $-\mathrm{H}$ \\
\hline 28 & $-\mathrm{H}$ & $-\mathrm{OCH}_{3}$ & $-\mathrm{H}$ & $-\mathrm{H}$ & $-\mathrm{H}$ & $-\mathrm{H}$ & $-\mathrm{OCH}_{3}$ & $-\mathrm{H}$ & $-\mathrm{H}$ & $-\mathrm{H}$ \\
\hline 29 & $-\mathrm{H}$ & $-\mathrm{H}$ & $-\mathrm{OCH}_{3}$ & $-\mathrm{H}$ & $-\mathrm{H}$ & $-\mathrm{H}$ & $-\mathrm{H}$ & $-\mathrm{OCH}_{3}$ & $-\mathrm{H}$ & $-\mathrm{H}$ \\
\hline 30 & $-\mathrm{OCH}_{3}$ & $-\mathrm{OCH}_{3}$ & $-\mathrm{H}$ & $-\mathrm{H}$ & $-\mathrm{H}$ & $-\mathrm{OCH}_{3}$ & $-\mathrm{OCH}_{3}$ & $-\mathrm{H}$ & $-\mathrm{H}$ & $-\mathrm{H}$ \\
\hline 31 & $-\mathrm{OCH}_{3}$ & $-\mathrm{H}$ & $-\mathrm{OCH}_{3}$ & $-\mathrm{H}$ & $-\mathrm{H}$ & $-\mathrm{OCH}_{3}$ & $-\mathrm{H}$ & $-\mathrm{OCH}_{3}$ & $-\mathrm{H}$ & $-\mathrm{H}$ \\
\hline 32 & $-\mathrm{OCH}_{3}$ & $-\mathrm{H}$ & $-\mathrm{H}$ & $-\mathrm{OCH}_{3}$ & $-\mathrm{H}$ & $-\mathrm{OCH}_{3}$ & $-\mathrm{H}$ & $-\mathrm{H}$ & $-\mathrm{OCH}_{3}$ & $-\mathrm{H}$ \\
\hline 33 & $-\mathrm{OCH}_{3}$ & $-\mathrm{H}$ & $-\mathrm{H}$ & $-\mathrm{H}$ & $-\mathrm{OCH}_{3}$ & $-\mathrm{OCH}_{3}$ & $-\mathrm{H}$ & $-\mathrm{H}$ & $-\mathrm{H}$ & $-\mathrm{OCH}_{3}$ \\
\hline 34 & $-\mathrm{H}$ & $-\mathrm{OCH}_{3}$ & $-\mathrm{OCH}_{3}$ & $-\mathrm{H}$ & $-\mathrm{H}$ & $-\mathrm{H}$ & $-\mathrm{OCH}_{3}$ & $-\mathrm{OCH}_{3}$ & $-\mathrm{H}$ & $-\mathrm{H}$ \\
\hline 35 & $-\mathrm{H}$ & $-\mathrm{OCH}_{3}$ & $-\mathrm{H}$ & $-\mathrm{OCH}_{3}$ & $-\mathrm{H}$ & $-\mathrm{H}$ & $-\mathrm{H}$ & $-\mathrm{OCH}_{3}$ & $-\mathrm{OCH}_{3}$ & $-\mathrm{H}$ \\
\hline 36 & $-\mathrm{OCH}_{3}$ & $-\mathrm{OCH}_{3}$ & $-\mathrm{OCH}_{3}$ & $-\mathrm{H}$ & $-\mathrm{H}$ & $-\mathrm{OCH}_{3}$ & $-\mathrm{OCH}_{3}$ & $-\mathrm{OCH}_{3}$ & $-\mathrm{H}$ & $-\mathrm{H}$ \\
\hline 37 & $-\mathrm{OCH}_{3}$ & $-\mathrm{OCH}_{3}$ & $-\mathrm{H}$ & $-\mathrm{H}$ & $-\mathrm{OCH}_{3}$ & $-\mathrm{OCH}_{3}$ & $-\mathrm{OCH}_{3}$ & $-\mathrm{H}$ & $-\mathrm{H}$ & $-\mathrm{OCH}_{3}$ \\
\hline 38 & $-\mathrm{OCH}_{3}$ & $-\mathrm{H}$ & $-\mathrm{OCH}_{3}$ & $-\mathrm{OCH}_{3}$ & & $-\mathrm{OCH}_{3}$ & $-\mathrm{OCH}_{3}$ & $-\mathrm{H}$ & $-\mathrm{OCH}_{3}$ & $-\mathrm{H}$ \\
\hline 39 & $-\mathrm{OCH}_{3}$ & $-\mathrm{H}$ & $-\mathrm{OCH}_{3}$ & $-\mathrm{H}$ & $-\mathrm{OCH}_{3}$ & $-\mathrm{OCH}_{3}$ & $-\mathrm{H}$ & $-\mathrm{OCH}_{3}$ & $-\mathrm{H}$ & $-\mathrm{OCH}_{3}$ \\
\hline 40 & $-\mathrm{H}$ & $-\mathrm{OCH}_{3}$ & $-\mathrm{OCH}_{3}$ & $-\mathrm{OCH}_{3}$ & $-\mathrm{H}$ & $-\mathrm{H}$ & $-\mathrm{OCH}_{3}$ & $-\mathrm{OCH}_{3}$ & $-\mathrm{OCH}_{3}$ & $-\mathrm{H}$ \\
\hline 41 & $-\mathrm{OCH}_{3}$ & $-\mathrm{OCH}_{3}$ & $-\mathrm{OCH}_{3}$ & $-\mathrm{OCH}_{3}$ & $-\mathrm{H}$ & $-\mathrm{OCH}_{3}$ & $-\mathrm{OCH}_{3}$ & $-\mathrm{OCH}_{3}$ & $-\mathrm{OCH}_{3}$ & $-\mathrm{H}$ \\
\hline 42 & $-\mathrm{H}$ & $-\mathrm{H}$ & $-\mathrm{H}$ & $-\mathrm{H}$ & $-\mathrm{H}$ & $-\mathrm{H}$ & $-\mathrm{OCH}_{3}$ & $-\mathrm{OCH}_{3}$ & $-\mathrm{OCH}_{3}$ & $-\mathrm{H}$ \\
\hline 43 & $-\mathrm{H}$ & $-\mathrm{H}$ & $-\mathrm{OCH}_{3}$ & $-\mathrm{H}$ & $-\mathrm{H}$ & $-\mathrm{H}$ & $-\mathrm{OCH}_{3}$ & $-\mathrm{OCH}_{3}$ & $-\mathrm{H}$ & $-\mathrm{H}$ \\
\hline 44 & $-\mathrm{H}$ & $-\mathrm{H}$ & $-\mathrm{OCH}_{3}$ & $-\mathrm{H}$ & $-\mathrm{H}$ & $-\mathrm{H}$ & $-\mathrm{OCH}_{3}$ & $-\mathrm{OCH}_{3}$ & $-\mathrm{OCH}_{3}$ & $-\mathrm{H}$ \\
\hline 45 & $-\mathrm{H}$ & $-\mathrm{OCH}_{3}$ & $-\mathrm{H}$ & $-\mathrm{H}$ & $-\mathrm{H}$ & $-\mathrm{H}$ & $-\mathrm{OCH}_{3}$ & $-\mathrm{OCH}_{3}$ & $-\mathrm{OCH}_{3}$ & $-\mathrm{H}$ \\
\hline 46 & $-\mathrm{H}$ & $-\mathrm{OCH}_{3}$ & $-\mathrm{OCH}_{3}$ & $-\mathrm{H}$ & $-\mathrm{H}$ & $-\mathrm{H}$ & $-\mathrm{OCH}_{3}$ & $-\mathrm{OCH}_{3}$ & $-\mathrm{OCH}_{3}$ & $-\mathrm{H}$ \\
\hline 47 & $-\mathrm{OCH}_{3}$ & $-\mathrm{OCH}_{3}$ & $-\mathrm{OCH}_{3}$ & $-\mathrm{H}$ & $-\mathrm{H}$ & $-\mathrm{H}$ & $-\mathrm{OCH}_{3}$ & $-\mathrm{OCH}_{3}$ & $-\mathrm{OCH}_{3}$ & $-\mathrm{H}$ \\
\hline 48 & $-\mathrm{OCH}_{3}$ & $-\mathrm{H}$ & $-\mathrm{OCH}_{3}$ & $-\mathrm{OCH}_{3}$ & $-\mathrm{H}$ & $-\mathrm{H}$ & $-\mathrm{OCH}_{3}$ & $-\mathrm{OCH}_{3}$ & $-\mathrm{OCH}_{3}$ & $-\mathrm{H}$ \\
\hline
\end{tabular}


Table 2. Compounds 49 - 68.

\begin{tabular}{|c|c|c|c|c|c|c|c|c|c|c|}
\hline Compounds & $\mathrm{R}_{1}$ & $\mathrm{R}_{2}$ & $\mathrm{R}_{3}$ & $\mathrm{R}_{4}$ & $\mathrm{R}_{5}$ & $\mathrm{R}_{6}$ & $\mathrm{R}_{7}$ & $\mathrm{R}_{8}$ & $\mathrm{R}_{9}$ & $\mathrm{R}_{10}$ \\
\hline 49 & $-\mathrm{H}$ & $-\mathrm{H}$ & $-\mathrm{OH}$ & & $-\mathrm{H}$ & $-\mathrm{H}$ & $-\mathrm{H}$ & $-\mathrm{OH}$ & $-\mathrm{H}$ & $-\mathrm{H}$ \\
\hline 50 & $-\mathrm{H}$ & $-\mathrm{H}$ & $-\mathrm{OH}$ & $-\mathrm{OCH}_{3}$ & $-\mathrm{H}$ & $-\mathrm{H}$ & $-\mathrm{H}$ & $-\mathrm{OH}$ & $-\mathrm{OCH}_{3}$ & $-\mathrm{H}$ \\
\hline 51 & $-\mathrm{H}$ & $-\mathrm{CH}_{3}$ & $-\mathrm{OH}$ & $-\mathrm{CH}_{3}$ & $-\mathrm{H}$ & $-\mathrm{H}$ & $-\mathrm{CH}_{3}$ & $-\mathrm{OH}$ & $-\mathrm{CH}_{3}$ & $-\mathrm{H}$ \\
\hline 52 & $-\mathrm{H}$ & $-\mathrm{C}_{2} \mathrm{H}_{5}$ & $-\mathrm{OH}$ & $-\mathrm{C}_{2} \mathrm{H}_{5}$ & $-\mathrm{H}$ & $-\mathrm{H}$ & $-\mathrm{C}_{2} \mathrm{H}_{5}$ & $-\mathrm{OH}$ & $-\mathrm{C}_{2} \mathrm{H}_{5}$ & $-\mathrm{H}$ \\
\hline 53 & $-\mathrm{H}$ & $\mathrm{t}-\mathrm{C}_{4} \mathrm{H}_{9}$ & $-\mathrm{OH}$ & $\mathrm{t}-\mathrm{C}_{4} \mathrm{H}_{9}$ & $-\mathrm{H}$ & $-\mathrm{H}$ & $\mathrm{t}-\mathrm{C}_{4} \mathrm{H}_{9}$ & $-\mathrm{OH}$ & $\mathrm{t}-\mathrm{C}_{4} \mathrm{H}_{9}$ & $-\mathrm{H}$ \\
\hline 54 & $-\mathrm{H}$ & $\mathrm{i}-\mathrm{C}_{3} \mathrm{H}_{7}$ & $-\mathrm{OH}$ & $\mathrm{i}-\mathrm{C}_{3} \mathrm{H}_{7}$ & $-\mathrm{H}$ & $-\mathrm{H}$ & $\mathrm{i}-\mathrm{C}_{3} \mathrm{H}_{7}$ & $-\mathrm{OH}$ & $\mathrm{i}-\mathrm{C}_{3} \mathrm{H}_{7}$ & $-\mathrm{H}$ \\
\hline 55 & $-\mathrm{H}$ & $-\mathrm{OCH}_{3}$ & $-\mathrm{OH}$ & $-\mathrm{OCH}_{3}$ & $-\mathrm{H}$ & $-\mathrm{H}$ & $-\mathrm{OCH}_{3}$ & $-\mathrm{OH}$ & $-\mathrm{OCH}_{3}$ & $-\mathrm{H}$ \\
\hline 56 & $-\mathrm{H}$ & $-\mathrm{Cl}$ & $-\mathrm{OH}$ & $-\mathrm{Cl}$ & $-\mathrm{H}$ & $-\mathrm{H}$ & $-\mathrm{Cl}$ & $-\mathrm{OH}$ & $-\mathrm{Cl}$ & $-\mathrm{H}$ \\
\hline 57 & $-\mathrm{H}$ & $-\mathrm{H}$ & $-\mathrm{OCH}_{2} \mathrm{CH}_{3}$ & $-\mathrm{H}$ & $-\mathrm{H}$ & $-\mathrm{H}$ & $-\mathrm{H}$ & $-\mathrm{OCH}_{2} \mathrm{CH}_{3}$ & $-\mathrm{H}$ & $-\mathrm{H}$ \\
\hline 58 & $-\mathrm{OCH}_{3}$ & $-\mathrm{OCH}_{3}$ & $-\mathrm{H}$ & $-\mathrm{H}$ & $-\mathrm{H}$ & $-\mathrm{OCH}_{3}$ & $-\mathrm{OCH}_{3}$ & $-\mathrm{H}$ & $-\mathrm{H}$ & $-\mathrm{H}$ \\
\hline 59 & $-\mathrm{OCH}_{3}$ & $-\mathrm{H}$ & $-\mathrm{H}$ & $-\mathrm{H}$ & $-\mathrm{H}$ & $-\mathrm{OCH}_{3}$ & $-\mathrm{H}$ & $-\mathrm{H}$ & $-\mathrm{H}$ & $-\mathrm{H}$ \\
\hline 60 & $-\mathrm{H}$ & $-\mathrm{OCH}_{3}$ & $-\mathrm{OCH}_{3}$ & $-\mathrm{OCH}_{3}$ & $-\mathrm{H}$ & $-\mathrm{H}$ & $-\mathrm{OCH}_{3}$ & $-\mathrm{OCH}_{3}$ & $-\mathrm{OCH}_{3}$ & $-\mathrm{H}$ \\
\hline 61 & $-\mathrm{H}$ & $-\mathrm{OCH}_{2} \mathrm{CH}=\mathrm{CH}_{2}$ & $-\mathrm{OCH}_{3}$ & $-\mathrm{H}$ & $-\mathrm{H}$ & $-\mathrm{H}$ & $-\mathrm{OCH}_{2} \mathrm{CH}=\mathrm{CH}_{2}$ & $-\mathrm{OCH}_{3}$ & $-\mathrm{H}$ & $-\mathrm{H}$ \\
\hline 62 & $-\mathrm{H}$ & $-\mathrm{H}$ & $-\mathrm{O}\left(\mathrm{CH}_{2}\right)_{3} \mathrm{~N}\left(\mathrm{CH}_{3}\right)_{2}$ & $-\mathrm{H}$ & $-\mathrm{H}$ & $-\mathrm{H}$ & $-\mathrm{H}$ & $-\mathrm{O}\left(\mathrm{CH}_{2}\right)_{3} \mathrm{~N}\left(\mathrm{CH}_{3}\right)_{2}$ & $-\mathrm{H}$ & $-\mathrm{H}$ \\
\hline 63 & $-\mathrm{H}$ & $-\mathrm{H}$ & $\begin{array}{c}\text {-(Tatrahydro-2H- } \\
\text { pyra-2-yl)oxy }\end{array}$ & $-\mathrm{H}$ & $-\mathrm{H}$ & $-\mathrm{H}$ & $-\mathrm{H}$ & $\begin{array}{c}\text {-(Tatrahydro-2H- } \\
\text { pyra-2-yl)oxy }\end{array}$ & $-\mathrm{H}$ & $-\mathrm{H}$ \\
\hline 64 & $-\mathrm{H}$ & $-\mathrm{H}$ & $-\mathrm{N}\left(\mathrm{CH}_{3}\right)_{2}$ & $-\mathrm{H}$ & $-\mathrm{H}$ & $-\mathrm{H}$ & $-\mathrm{H}$ & $-\mathrm{N}\left(\mathrm{CH}_{3}\right)_{2}$ & $-\mathrm{H}$ & $-\mathrm{H}$ \\
\hline 65 & $-\mathrm{H}$ & $-\mathrm{H}$ & $-F$ & $-\mathrm{H}$ & $-\mathrm{H}$ & $-\mathrm{H}$ & $-\mathrm{H}$ & $-F$ & $-\mathrm{H}$ & $-\mathrm{H}$ \\
\hline 66 & $-\mathrm{Br}$ & $-\mathrm{H}$ & $-\mathrm{H}$ & $-\mathrm{H}$ & $-\mathrm{H}$ & $-\mathrm{Br}$ & $-\mathrm{H}$ & $-\mathrm{H}$ & $-\mathrm{H}$ & $-\mathrm{H}$ \\
\hline 67 & $-\mathrm{Cl}$ & $-\mathrm{H}$ & $-\mathrm{H}$ & $-\mathrm{H}$ & $-\mathrm{H}$ & $-\mathrm{Cl}$ & $-\mathrm{H}$ & $-\mathrm{H}$ & $-\mathrm{H}$ & $-\mathrm{H}$ \\
\hline 68 & $-\mathrm{CF}_{3}$ & $-\mathrm{H}$ & $-\mathrm{H}$ & $-\mathrm{H}$ & $-\mathrm{H}$ & $-\mathrm{CF}_{3}$ & $-\mathrm{H}$ & $-\mathrm{H}$ & $-\mathrm{H}$ & $-\mathrm{H}$ \\
\hline
\end{tabular}

Table 3. Compounds 69 - 92.

\begin{tabular}{|c|c|c|c|c|c|c|c|c|c|c|}
\hline & & & $\mathrm{R}_{4}$ & & & 1 & & & & \\
\hline Compounds & $\mathrm{R}_{1}$ & $\mathrm{R}_{2}$ & $\mathrm{R}_{3}$ & $\mathrm{R}_{4}$ & $\mathrm{R}_{5}$ & $\mathrm{R}_{6}$ & $\mathrm{R}_{7}$ & $\mathrm{R}_{8}$ & $\mathrm{R}_{9}$ & $\mathrm{R}_{10}$ \\
\hline 69 & $-\mathrm{H}$ & $-\mathrm{H}$ & $-\mathrm{OH}$ & $-\mathrm{H}$ & $-\mathrm{H}$ & $-\mathrm{H}$ & $-\mathrm{H}$ & $-\mathrm{OH}$ & $-\mathrm{H}$ & $-\mathrm{H}$ \\
\hline 70 & $-\mathrm{H}$ & $-\mathrm{CH}_{3}$ & $-\mathrm{OH}$ & $-\mathrm{CH}_{3}$ & $-\mathrm{H}$ & $-\mathrm{H}$ & $-\mathrm{CH}_{3}$ & $-\mathrm{OH}$ & $-\mathrm{CH}_{3}$ & $-\mathrm{H}$ \\
\hline 71 & $-\mathrm{H}$ & $-\mathrm{H}$ & $-\mathrm{OH}$ & $-F$ & $-\mathrm{H}$ & $-\mathrm{H}$ & $-\mathrm{H}$ & $-\mathrm{OH}$ & $-F$ & $-\mathrm{H}$ \\
\hline 72 & $-\mathrm{H}$ & $-\mathrm{H}$ & $-\mathrm{OH}$ & $-\mathrm{OCH}_{3}$ & $-\mathrm{H}$ & $-\mathrm{H}$ & $-\mathrm{H}$ & $-\mathrm{OH}$ & $-\mathrm{OCH}_{3}$ & $-\mathrm{H}$ \\
\hline 73 & $-\mathrm{H}$ & $-\mathrm{C}_{2} \mathrm{H}_{5}$ & $-\mathrm{OH}$ & $-\mathrm{C}_{2} \mathrm{H}_{5}$ & $-\mathrm{H}$ & $-\mathrm{H}$ & $-\mathrm{C}_{2} \mathrm{H}_{5}$ & $-\mathrm{OH}$ & $-\mathrm{C}_{2} \mathrm{H}_{5}$ & $-\mathrm{H}$ \\
\hline 74 & $-\mathrm{H}$ & $\mathrm{i}-\mathrm{C}_{3} \mathrm{H}_{7}$ & $-\mathrm{OH}$ & $\mathrm{i}-\mathrm{C}_{3} \mathrm{H}_{7}$ & $-\mathrm{H}$ & $-\mathrm{H}$ & $\mathrm{i}-\mathrm{C}_{3} \mathrm{H}_{7}$ & $-\mathrm{OH}$ & $\mathrm{i}-\mathrm{C}_{3} \mathrm{H}_{7}$ & $-\mathrm{H}$ \\
\hline 75 & $-\mathrm{H}$ & $\mathrm{t}-\mathrm{C}_{4} \mathrm{H}_{9}$ & $-\mathrm{OH}$ & $\mathrm{t}-\mathrm{C}_{4} \mathrm{H}_{9}$ & $-\mathrm{H}$ & $-\mathrm{H}$ & $\mathrm{t}-\mathrm{C}_{4} \mathrm{H}_{9}$ & $-\mathrm{OH}$ & $\mathrm{t}-\mathrm{C}_{4} \mathrm{H}_{9}$ & $-\mathrm{H}$ \\
\hline 76 & $-\mathrm{H}$ & $-\mathrm{OCH}_{3}$ & $-\mathrm{OH}$ & $-\mathrm{OCH}_{3}$ & $-\mathrm{H}$ & $-\mathrm{H}$ & $-\mathrm{OCH}_{3}$ & $-\mathrm{OH}$ & $-\mathrm{OCH}_{3}$ & $-\mathrm{H}$ \\
\hline 77 & $-\mathrm{OH}$ & $-\mathrm{H}$ & $-\mathrm{H}$ & $-\mathrm{H}$ & $-\mathrm{H}$ & $-\mathrm{OH}$ & $-\mathrm{H}$ & $-\mathrm{H}$ & $-\mathrm{H}$ & $-\mathrm{H}$ \\
\hline 78 & $-F$ & $-\mathrm{H}$ & $-\mathrm{H}$ & $-\mathrm{H}$ & $-\mathrm{H}$ & $-F$ & $-\mathrm{H}$ & $-\mathrm{H}$ & $-\mathrm{H}$ & $-\mathrm{H}$ \\
\hline 79 & $-\mathrm{H}$ & $-\mathrm{OCH}_{3}$ & $-\mathrm{OCH}_{3}$ & $-\mathrm{H}$ & $-\mathrm{H}$ & $-\mathrm{H}$ & $-\mathrm{OCH}_{3}$ & $-\mathrm{OCH}_{3}$ & $-\mathrm{H}$ & $-\mathrm{H}$ \\
\hline 80 & $-\mathrm{H}$ & $-\mathrm{OCH}_{3}$ & $-\mathrm{OH}$ & $-\mathrm{H}$ & $-\mathrm{H}$ & $-\mathrm{H}$ & $-\mathrm{OCH}_{3}$ & $-\mathrm{OH}$ & $-\mathrm{H}$ & $-\mathrm{H}$ \\
\hline 81 & $-\mathrm{H}$ & $-\mathrm{H}$ & $-\mathrm{OCH}_{2} \mathrm{CH}_{3}$ & $-\mathrm{H}$ & $-\mathrm{H}$ & $-\mathrm{H}$ & $-\mathrm{H}$ & $-\mathrm{OCH}_{2} \mathrm{CH}_{3}$ & $-\mathrm{H}$ & $-\mathrm{H}$ \\
\hline 82 & $-\mathrm{H}$ & $-\mathrm{OCH}_{3}$ & $-\mathrm{OCH}_{3}$ & $-\mathrm{OCH}_{3}$ & $-\mathrm{H}$ & $-\mathrm{H}$ & $-\mathrm{OCH}_{3}$ & $-\mathrm{OCH}_{3}$ & $-\mathrm{OCH}_{3}$ & $-\mathrm{H}$ \\
\hline 83 & $-\mathrm{H}$ & $-\mathrm{H}$ & $-\mathrm{OCH}_{2} \mathrm{CH}=\mathrm{CH}_{2}$ & $-\mathrm{H}$ & $-\mathrm{H}$ & $-\mathrm{H}$ & $-\mathrm{H}$ & $-\mathrm{OCH}_{2} \mathrm{CH}=\mathrm{CH}_{2}$ & $-\mathrm{H}$ & $-\mathrm{H}$ \\
\hline 84 & $-\mathrm{H}$ & $-\mathrm{OCH}_{3}$ & $-\mathrm{OCH}_{2} \mathrm{CH}=\mathrm{CH}_{2}$ & $-\mathrm{H}$ & $-\mathrm{H}$ & $-\mathrm{H}$ & $-\mathrm{OCH}_{3}$ & $-\mathrm{OCH}_{2} \mathrm{CH}=\mathrm{CH}_{2}$ & $-\mathrm{H}$ & $-\mathrm{H}$ \\
\hline 85 & $-\mathrm{H}$ & $-\mathrm{H}$ & -(Tetrahydro-2H-pyran-yl)oxy & $-\mathrm{H}$ & $-\mathrm{H}$ & $-\mathrm{H}$ & $-\mathrm{H}$ & -(Tetrahydro-2H-pyran-yl)oxy & $-\mathrm{H}$ & $-\mathrm{H}$ \\
\hline 86 & $-\mathrm{CH}_{3}$ & $-\mathrm{H}$ & $-\mathrm{H}$ & $-\mathrm{CH}_{3}$ & $-\mathrm{H}$ & $-\mathrm{CH}_{3}$ & $-\mathrm{H}$ & $-\mathrm{H}$ & $-\mathrm{CH}_{3}$ & $-\mathrm{H}$ \\
\hline 87 & $-\mathrm{H}$ & $-\mathrm{H}$ & $-\mathrm{C}\left(\mathrm{CH}_{3}\right)_{3}$ & $-\mathrm{H}$ & $-\mathrm{H}$ & $-\mathrm{H}$ & $-\mathrm{H}$ & $-\mathrm{C}\left(\mathrm{CH}_{3}\right)_{3}$ & $-\mathrm{H}$ & $-\mathrm{H}$ \\
\hline 88 & $-\mathrm{H}$ & $-\mathrm{H}$ & $-F$ & $-\mathrm{H}$ & $-\mathrm{H}$ & $-\mathrm{H}$ & $-\mathrm{H}$ & $-F$ & $-\mathrm{H}$ & $-\mathrm{H}$ \\
\hline 89 & $-\mathrm{H}$ & $-\mathrm{Br}$ & $-\mathrm{H}$ & $-\mathrm{H}$ & $-\mathrm{H}$ & $-\mathrm{H}$ & $-\mathrm{Br}$ & $-\mathrm{H}$ & $-\mathrm{H}$ & $-\mathrm{H}$ \\
\hline 90 & $-\mathrm{Br}$ & $-\mathrm{H}$ & $-\mathrm{H}$ & $-\mathrm{H}$ & $-\mathrm{H}$ & $-\mathrm{Br}$ & $-\mathrm{H}$ & $-\mathrm{H}$ & $-\mathrm{H}$ & $-\mathrm{H}$ \\
\hline 91 & $-\mathrm{Cl}$ & $-\mathrm{H}$ & $-\mathrm{H}$ & $-\mathrm{H}$ & $-\mathrm{H}$ & $-\mathrm{Cl}$ & $-\mathrm{H}$ & $-\mathrm{H}$ & $-\mathrm{H}$ & $-\mathrm{H}$ \\
\hline 92 & $-\mathrm{CF}_{3}$ & $-\mathrm{H}$ & $-\mathrm{H}$ & $-\mathrm{H}$ & $-\mathrm{H}$ & $-\mathrm{CF}_{3}$ & $-\mathrm{H}$ & $-\mathrm{H}$ & $-\mathrm{H}$ & $-\mathrm{H}$ \\
\hline
\end{tabular}


Table 4. Compounds 93 - 110.

\begin{tabular}{|c|c|c|c|c|c|c|c|}
\hline Compounds & $\mathrm{R}_{1}$ & $\mathrm{R}_{2}$ & $\mathrm{R}_{3}$ & Compounds & $\mathrm{R}_{1}$ & $\mathrm{R}_{2}$ & $\mathrm{R}_{3}$ \\
\hline 93 & $2-\mathrm{OH}$ & $2-\mathrm{OH}$ & $-\mathrm{CH}_{3}$ & 102 & $2-\mathrm{F}$ & $2-\mathrm{F}$ & $-\mathrm{H}$ \\
\hline 94 & $4-\mathrm{OH}$ & $4-\mathrm{OH}$ & $-\mathrm{CH}_{3}$ & 103 & $4-\mathrm{OCH}_{3}$ & $4-\mathrm{OCH}_{3}$ & $-\mathrm{CH}_{3}$ \\
\hline 95 & $4-\mathrm{OH} ; 5-\mathrm{OC}_{2} \mathrm{H}_{5}$ & $4-\mathrm{OH} ; 5-\mathrm{OC}_{2} \mathrm{H}_{5}$ & $-\mathrm{CH}_{3}$ & 104 & $4-\mathrm{OH} ; 5-\mathrm{OC}_{2} \mathrm{H}_{5}$ & $4-\mathrm{OH} ; 5-\mathrm{OC}_{2} \mathrm{H}_{5}$ & $-\mathrm{C}_{2} \mathrm{H}_{5}$ \\
\hline 96 & $4-\mathrm{OCH}_{3}$ & $4-\mathrm{OCH}_{3}$ & $-\mathrm{C}_{2} \mathrm{H}_{5}$ & 105 & $4-\mathrm{OH}$ & $4-\mathrm{OH}$ & $-\mathrm{C}_{2} \mathrm{H}_{5}$ \\
\hline 97 & $4-\mathrm{OH}$ & $4-\mathrm{OH}$ & $-\mathrm{C}_{3} \mathrm{H}_{7}$ & 106 & $-\mathrm{H}$ & $-\mathrm{H}$ & $-\mathrm{H}$ \\
\hline 98 & $2-\mathrm{F}$ & $2-\mathrm{F}$ & $-\mathrm{H}$ & 107 & $2-\mathrm{Cl}$ & $2-\mathrm{Cl}$ & $-\mathrm{H}$ \\
\hline 99 & $2-\mathrm{Br}$ & $2-\mathrm{Br}$ & $-\mathrm{H}$ & 108 & $2-\mathrm{NO}_{2}$ & $2-\mathrm{NO}_{2}$ & $-\mathrm{H}$ \\
\hline 100 & $3-\mathrm{Cl}$ & $3-\mathrm{Cl}$ & $-\mathrm{H}$ & 109 & $4-\mathrm{F}$ & $4-\mathrm{F}$ & $-\mathrm{H}$ \\
\hline 101 & $4-\mathrm{Cl}$ & $4-\mathrm{Cl}$ & $-\mathrm{H}$ & 110 & $4-\mathrm{N}\left(\mathrm{CH}_{3}\right)_{3}$ & $4-\mathrm{N}\left(\mathrm{CH}_{3}\right)_{3}$ & $-\mathrm{H}$ \\
\hline
\end{tabular}

compound, a strong electron-donating moiety may remove the bioactivity and a strong electron-withdrawing one may reduce it; 3) The acetone and cyclohexanone spacers are much more favorable than the cyclopentanone one; 4) The introduction of heteroaromatic ring is also pharmacologically accessible. And the following conclusions about SAR were drawn by the the research group of H. Yamakoshi; 5) Bis(aryl methylidene) acetone serves as the most promising skeleton for eliciting cytotoxicity; 6) The 3-oxo-1,4-pentadiene structure is essential for eliciting cytotoxicity; 7) Hexa-substituted compounds exhibit strong activities; 8) 3,4,5-Hexasubstitution results in the highest potency; 9) The symmetry between two aryl rings is important for tetra-substituted analogues but not a requirement for hexa-substituted analogues; 10) para-positions are allowed to introduce of additional functional groups for use as molecular probes. From the SAR conclusions, they have obtained several potential anticancer compounds.

\section{Conclusion}

The anticancer activity which has been confirmed in curcumin, were also been found in those mono-carbonyl curcumin analogues. Some of compounds in the class of mono-carbonyl curcumin analogues possess even better activity than curcumin. In addition, the class of monocarbonyl curcumin analogues overcome the drawback of poor stability in curcumin and have a simple synthetic routes compared with curcumin. Having these advantages, the mono-carbonyl curcumin analogues may be the "super curcumin" for which we are research. So it is meaningful to do further researches on the mono-carbonyl curcumin analogues. What more, some other pharmacological activity were also been found in those mono-carbonyl curcumin analogues. such as antioxidant [64-66], anti-inflammatory [67,68], anti-bacterial [69], anti-AD [70], with which the class of compounds will having good development prospects.

\section{Acknowledgements}

This research was supported by the National Natural Science Foundation of China (No. 81273537), Scientific Research Fund of Hunan Provincial Education Department (No. 12K095), and the Key Project of Ministry of Education of the People's Republic of China (No. 20809).

\section{REFERENCES}

[1] I. Rahman, S. K. Biswas and P. A. Kirkham, "Regulation of Inflammation and Redoxsignaling by Dietarypolyphenols", Biochemical Pharmacology, Vol. 72, No. 11, 2006, pp. 1439-1452. doi:10.1016/j.bcp.2006.07.004

[2] N. Tirkey, G. Kaur, G. Vij and K. Chopra, "Curcumin, a Diferuloylmethane, Attenuates Cyclosporine-Induced Renal Dysfunction and Oxidative Stress in Rat Kidneys," BMC Pharmacology, Vol. 5, No. 15, 2005. doi:10.1186/1471-2210-5-15

[3] B. V. Reddy, J. S. Sundary, E. Balamurugan and V. P. Menon, "Prevention of Nicotine and Streptozotocin Treatment Induced Circulatory Oxidative Stress by Bis1,7-(2-hydroxyphenyl)-hepta-1,6-diene-3,5-dione in Diabetic Rats," Molecular and Cellular Biochemistry, Vol. 331, No. 1-2, 2009, pp. 127-133. 
doi:10.1007/s11010-009-0150-1

[4] D. K. Agrawal and P. K. Mishra, "Curcumin and Its Analogues: Potential Anticancer Agents," Medicinal Research Reviews, Vol. 30, No. 5, 2010, pp. 818-860. doi:10.1002/med.20188

[5] P. Basneta and N. Skalko-Basnet, "Curcumin: An AntiInflammatory Molecule from a Curry Spice on the Path to Cancer Treatment," Molecules, Vol. 16, No. 6, 2011, pp. 4567-4598. doi:10.3390/melecules 16064567

[6] H. S. Na, M. H. Cha, D. R. Oh, C. W. Cho, J. H. Rhee and Y. R. Kim, "Protective Mechanism of Curcumin against Vibrio vulnificus Infection," FEMS Immunology \& Medical Microbiology, Vol. 63, No. 3, 2011, pp. 355362. doi:10.1111/j.1574-695X.2011.00855.x

[7] A. Goel, A. B. Kunnumakkara and B. B. Aggarwal, "Curcumin as 'Curecumin': From Kitchen to Clinic," Biochemical Pharmacology, Vol. 75, No. 4, 2008, pp. 787-809. doi:10.1016/j.bcp.2007.08.016

[8] R. A. Sharma, W. P. Steward and A. J. Gescher, "Pharmacokinetics and Pharmacodynamics of Curcumin," $A d$ vances in Experimental Medicine and Biology, Vol. 595, 2007, pp. 453-470. doi:10.1007/978-0-387-46401-5 20

[9] M. H. Pan, T. M. Huang and J. K. Lin, "Biotransformation of Curcumin through Reduction and Glucuronidation in mice," Drug Metabolism and Disposition, Vol. 27, No. 4, 1999, pp. 486-494. doi:0090-9556/99/2701-0486-494\$02.00/0

[10] D. Youssef, C. E. Nichols, T. S. Cameron, J. Balzarini, E. D. Clercq and A. Jha, "Design, Synthesis, and Cytostatic Activity of Novel Cyclic Curcumin Analogues," Bioorganic \& Medicinal Chemistry Letters, Vol. 17, No. 20, 2007, pp. 5624-5629. doi:10.1016/j.bmcl.2007.07.079

[11] X. Qiu, Z. Liu, W. Y. Shao, X. Liu, D. P. Jing, Y. J. Yu, L. K. An, S. L.Huang, X. Z. Bu, Z. S. Huang and L. Q. $\mathrm{Gu}$, "Synthesis and Evaluation of Curcumin Analogues as Potential Thioredoxin Reductase Inhibitors," Bioorganic \& Medicinal Chemistry, Vol. 16, No. 17, 2008, pp. 80358041. doi:10.1016/j.bmc.2008.07.054

[12] Q. Zhang, Y. Zhong, L. N. Yan, X. Sun, T. Gong and Z. R. Zhang, "Synthesis and Preliminary Evaluation of Curcumin Analogues as Cytotoxic Agents," Bioorganic \& Medicinal Chemistry Letters, Vol. 21, No. 3, 2011, pp. 1010-1014. doi:10.1016/j.bmcl.2010.12.020

[13] S. K. Dubey, A. K. Sharma, U. Narain, K. Misra and U. Pati, "Design, Synthesis and Characterization of Some Bioactive Conjugates of Curcumin with Glycine, Glutamic Acid, Valine and Demethylenated Piperic Acid and Study of Their Antimicrobial and Antiproliferative Properties," European Journal of Medicinal Chemistry, Vol. 43, No. 9, 2008, pp. 1837-1846. doi:10.1016/j.ejmech.2007.11.027

[14] L. Lin, Q. Shi, A. K. Nyarko, K. F. Bastow, C. C. Wu, C. Y. Su, C. C. Y Shih and K. H. Lee, "Antitumor Agents. 250. Design and Synthesis of New Curcumin Analogues as Potential Anti-Prostate Cancer Agents," Journal of Medicinal Chemistry, Vol. 49, No. 13, 2006, pp. 39633972. doi:10.1021/jm051043z

[15] J. A. Lenhart, X. Ling, R. Gandhi, T. L. Guo, P. M. Gerk, D. H. Brunzell and S. Zhang, "'Clicked' Bivalent Ligands
Containing Curcumin and Cholesterol as Multifunctional Abeta Oligomerization Inhibitors: Design, Synthesis, and Biological Characterization," Journal of Medicinal Chemistry, Vol. 53, No. 16, 2010, pp. 6198-6209. doi:10.1021/jm100601q

[16] J. Zhou, G. Geng, Q. Shi, F. Sauriol and J. H. Wu, "Design and Synthesis of Androgen Receptor Antagonists with Bulky Side Chains for Overcoming Antiandrogen Resistance," Journal of Medicinal Chemistry, Vol. 52, No. 17, 2009, pp. 5546-5550. doi:10.1021/jm801218k

[17] Y. J. Wang, M. H. Pan, A. L. Cheng, L. I. Lin, Y. S. Ho, C. Y. Hsieh and J. K. Lin, "Stability of Curcumin in Buffer Solutions and Characterization of Its Degradation Products," Journal of Pharmaceutical and Biomedical Analysis, Vol. 15, No. 12, 1997, pp. 1867-1876. doi:10.1016/S0731-7085(96)02024-9

[18] M. A. Tomren, M. Masson, T. Loftsson and H. H. Tønnesen, "Studies on Curcumin and Curcuminoids XXXI. Symmetric and Asymmetric Curcuminoids: Stability, Activity and Complexation with Cyclodextrin," International Journal of Pharmaceutics, Vol. 338, No. 1-2, 2007, pp. 27-34. doi:10.1016/j.ijpharm.2007.01.013

[19] G. D. Straganz, A. Glieder, L. Brecker, D. W. Ribbons and W. Steiner, "Acetylacetone-Cleaving Enzyme Dke: A Novel C-C-Bond-Cleaving Enzyme from Acinetobacter Johnsonii," Biochemical Journal, Vol. 369, No. 3, 2003, pp. 573-581. doi:10.1042/BJ20021047

[20] M. J. C. Rosemonda, L. S. J. Williamsa, T. Yamaguchib, T. Fujishitac and J. S. Walsha, "Enzymology of a Carbonyl Reduction Clearance Pathway for the HIV Integrase Inhibitor, S-1360: Role of Human Liver Cytosolic Aldo-Keto Reductases," Chemico-Biological Interactions, Vol. 147, No. 2, 2004, pp. 129-139.

doi:10.1016/j.cbi.2003.12.001

[21] G. Grogan, "Emergent Mechanistic Diversity of EnzymeCatalysed $\beta$-Diketone Cleavage," Biochemical Journal, Vol. 388, No. 3, 2005, pp. 721-730. doi:10.1042/BJ20042038

[22] G. Liang, L. Shao, Y. Wang, C. Zhao, Y. Chu, J. Xiao, Y. Zhao, X. Li and S. Yang, "Exploration and Synthesis of Curcumin Analogues with Improved Structural Stability Both in Vitro and in Vivo as Cytotoxic Agents," Bioorganic \& Medicinal Chemistry, Vol. 17, No. 6, 2009, pp. 2623-2631. doi:10.1016/j.bmc.2008.10.044

[23] T. C. Hour, J. Chen, C. Y. Huang, J. Y. Guan, S. H. Lu and Y. S. Pu, "Curcumin Enhances Cytotoxicity of Chemotherapeutic Agents in Prostate Cancer Cells by Inducing $\mathrm{p} 21^{\mathrm{WAF} 1 / \mathrm{CIP} 1}$ and C/EBP $\beta$ Expressions and Suppressing NF- $\mathrm{B}$ Activation," The Prostate, Vol. 51, No. 3, 2002, pp. 211-218. doi:10.1002/pros.10089

[24] M. M. Chan, D. Fong, K. J. Soprano, W. F. Holmes and H. Heverling, "Inhibition of Growth and Sensitization to Cisplatin-Mediated Killing of Ovarian Cancer Cells by Polyphenolic Chemopreventive Agents," Journal of Cellular Physiology, Vol. 194, No. 1, 2003, pp. 63-70. doi:10.1002/jep.10186

[25] J. Y. Koo, H. J. Kim, K. O. Jung and K. Y. Park, "Curcumin Inhibits the Growth of AGS Human Gastric Carcinoma Cells In Vitro and Shows Synergism with 5-Fluorouracil," Journal of Medicinal Food, Vol. 7, No. 2, 
2004, pp. 117-121. doi:10.1089/1096620041224229

[26] M. Notarbartolo, P. Poma, D. Perri, L. Dusonchet, M. Cervello and N. D'Alessandro, "Antitumoreffects of Curcumin, Alone or in Combination with Cisplatin or Doxorubicin, on Humanhepaticcancer Cells. Analysis of Their Possiblerelationship to Changes in NF-kB Activation Levels and in IAP Gene Expression," Cancer Letters, Vol. 224, No. 1, 2005, pp. 53-65. doi:10.1016/j.canlet.2004.10.051

[27] S. V. Bava, V. T. Puliappadamba, A. Deepti, A. Nair, D. Karunagaran and R. J. Anto, "Sensitization of Taxol-Induced Apoptosis by Curcumin Involves Down-Regulation of Nuclear Factor- $\kappa \mathrm{B}$ and the Serine/Threonine Kinase Akt and Is Independent of Tubulin Polymerization," The Journal of Biological Chemistry, Vol. 280, No. 8, 2005, pp. 6031-6038. doi:10.1074/jbc.M410647200

[28] B. B. Aggarwal, S. Shishodia, Y. Takada, S. Banerjee, R. A. Newman, C. E. Bueso-Ramos and J. E. Price, "Curcumin Suppresses the Paclitaxel-Induced Nuclear Factor- $\mathrm{KB}$ Pathway in Breast Cancer Cells and Inhibits Lung Metastasis of Human Breast Cancer in Nude Mice," Clinical Cancer Research, Vol. 11, No. 20, 2005, pp. 74907498. doi:10.1158/1078-0432.CCR-05-1192

[29] S. L. Ari , L. Strier, D. Kazanov, L. M. Shapiro, H. D. Sobol, I. Pinchuk, B. Marian, D. Lichtenberg and N. Arber, "Celecoxib and Curcumin Synergistically Inhibit the Growth of Colorectal Cancer Cells," Clinical Cancer Research, Vol. 11, 2005, pp. 6738-6744. doi:10.1158/1078-0432.CCR-05-0171

[30] S. Sen, H. Sharma and N. Singh, "Curcumin enhances Vinorelbine mediated apoptosis in NSCLC cells by the mitochondrial pathway," Biochemical and Biophysical Research Communications, Vol. 331, No. 4, 2005, pp. 1245-1252. doi:10.1016/j.bbrc.2005.04.044

[31] A. M. Kamat, G. Sethi and B. B. Aggarwal, "Curcumin Potentiates the Apoptotic Effects of Chemotherapeutic Agents and Cytokines through Down-Regulation of $\mathrm{Nu}-$ clear Factor- $\kappa \mathrm{B}$ and Nuclear Factor- $\kappa \mathrm{B}-$ Regulated Gene Products in IFN- $\alpha$-Sensitive and IFN- $\alpha$-Resistant Human Bladder Cancer Cells," Molecular Cancer Therapeutics, Vol. 6, No. 3, 2007, pp. 1022-1030. doi:10.1158/1535-7163.MCT-06-0545

[32] S. Lev-Ari, A. Vexler, A. Starr, M. Ashkenazy-Voghera, J. Greif, D. Aderka and R. Ben-Yosef, "Curcumin Augments Gemcitabine Cytotoxic Effect on Pancreatic Adenocarcinoma Cell Lines," Cancer Investigation, Vol. 25, No. 6, 2007, pp. 411-418. doi:10.1080/07357900701359577

[33] R. Kuttan, P. C. Sudheeran and C. D. Josph, "Turmeric and Curcumin as Topical Agents in Cancer Therapy," Tumori, Vol. 73, No. 1, 1987, pp. 29-31.

[34] R. A. Sharma, H. R. McLelland, K. A. Hill, C. R. Ireson, S. A. Euden, M. M. Manson, M. Pirmohamed, L. J. Marnett, A. J. Gescher and W. P. Steward, "Pharmacodynamic and Pharmacokinetic Study of Oral Curcuma Extract in Patients with Colorectal Cancer," Clinical Cancer Research, Vol. 7, No. 7, 2001, pp. 1894-1900.

[35] N. Dhillon, B. B. Aggarwal, R. A. Newman, R. A. Wolff, A. B. Kunnumakkara, J. L. Abbruzzese, C. S. Ng, V. Badmaev and R. Kurzrock, "Phase II Trial of Curcumin in Patients with Advanced Pancreatic Cancer," Clinical Cancer Research, Vol. 14, 2008, pp. 4491-4499. doi:10.1158/1078-0432.CCR-08-0024

[36] M. B. Robert, F. Kwiatowski, M. Leheurteur, F. Gachon, E. Planchat, C. Abrial, M. A. Mouret-Reynier, X. Durando, C. Barthomeuf and P. Chollet, "Phase I Dose Escalation Trial of Docetaxel Plus Curcumin in Patients with Advanced and Metastatic Breast Cancer," Cancer Biology \& Therapy, Vol. 9, No. 1, 2010, pp. 8-14. doi:10.4161/cbt.9.1.10392

[37] H. Ide, S. Tokiwa, K. Sakamaki, K. Nishio, S. Isotani, S. Muto, T. Hama, H. Masuda and S. Horie, "Combined Inhibitory Effects of Soy Isoflavones and Curcumin on the Production of Prostate-Specific Antigen," The Prostate, Vol. 70, No. 10, 2010, pp. 1127-1133. doi:10.1002/pros. 21147

[38] T. Golombick, T. H. Diamond, V. Badmaev, A. Manoharan and R. Ramakrishna, "The Potential Role of Curcumin in Patients with Monoclonal Gammopathy of Undefined Significance-Its Effect on Paraproteinemia and the Urinary N-Telopeptide of Type I Collagen Bone Turnover Marker," Clinical Cancer Research, Vol. 15, No. 18, 2009, pp. 5917-5922. doi:10.1158/1078-0432.CCR-08-2217

[39] K. Polasa, T. C. Raghuram, T. P. Krishna and K. Krishnaswamy, "Effect of Turmeric on Urinary Mutagens in Smokers," Mutagenesis, Vol. 7, No. 2, 1992, pp. 107-109. doi:10.1093/mutage/7.2.107

[40] A. L. Cheng, C. H. Hsu, J. K. Lin, M. M. Hsu, Y. F. Ho, T. S. Shen, J. Y. Ko, J. T. Lin, B. R. Lin, W. MingShiang, H. S. Yu, S. H. Jee, G. S. Chen, T. M. Chen, C. A. Chen, M. K. Lai, Y. S. Pu, M. H. Pan, Y. J. Wang, C. C. Tsai and C. Y. Hsieh, "Phase I Clinical Trial of Curcumin, a Chemopreventive Agent, in Patients with High-Risk or Pre-Malignant Lesions," Anticancer Research, Vol. 21, No. 4B, 2001, pp. 2895-2900.

[41] B. Rai, J. Kaur, R. Jacobs and J. Singh, "Possible Action Mechanism for Curcumin in Pre-Cancerous Lesions Based on Serum and Salivary Markers of Oxidative Stress," Journal of Oral Science, Vol. 52, No. 2, 2011, pp. 251-256. doi:10.2334/josnusd.52.251

[42] B. Wahlström and G. Blennow, "A Study on the Fate of Curcumin in the Rat," Acta Pharmacologica et Toxicologica, Vol. 43, No. 2, 1978, pp. 86-92. doi:10.1111/j.1600-0773.1978.tb02240.x

[43] M. H. Pan, T. M. Huang and J. K. Lin, "Biotransformation of Curcumin Through Reduction and Glucuronidation in Mice," Drug Metabolism and Disposition, Vol. 27, No. 4, 1999, pp. 486-494.

[44] S. S. Sardjiman, M. S. Reksohadiprodjo, L. Hakim, H. Goot and H. Timmerman, "1,5-Diphenyl-1,4-pentadiene3-ones and Cyclic Analogues as Antioxidative Agents. Synthesis and Structure-Activity Relationship," European Journal of Medicinal Chemistry, Vol. 32, No. 7-8, 1997, pp. 625-630. doi:10.1016/S0223-5234(97)83288-6

[45] Z. Du, R. Liu, W. Shao, X. Mao, L. Ma, L. Gu, Z. Huang and A. S. C. Chan, " $\alpha$-Glucosidase Inhibition of Natural Curcuminoids and Curcumin Analogs," European Journal of Medicinal Chemistry, Vol. 41, No. 2, 2006, pp. 213-218. doi:10.1016/j.ejmech.2005.10.012 
[46] K. Lee, F. H. A. Aziz, A. Syahida, F. Abas, K. Shaari, D. A. Israf and N. H. Lajis, "Synthesis and Biological Evaluation of Curcumin-Like Diarylpentanoid Analogues for Anti-Inflammatory, Antioxidant and Anti-Tyrosinase Activities," European Journal of Medicinal Chemistry, Vol. 44, No. 8, 2009, pp. 3195-3200. doi:10.1016/i.ejmech.2009.03.020

[47] G. Liang, X. Li, L. Chen, S. Yang, X. Wu, E. Studer, E. Gurley, P. B. Hylemon, F. Ye, Y. Li and H. Zhou, "Synthesis and Anti-Inflammatory Activities of Mono-Carbonyl Analogues of Curcumin," Bioorganic \& Medicinal Chemistry Letters, Vol. 18, No. 4, 2008, pp. 1525-1529. doi:10.1016/j.bmcl.2007.12.068

[48] J. A. Quincoces Suarez, D. G. Rando, R. P. Santos, C. P. Gonçalves, E. Ferreira, J. E. Carvalho, L. Kohn, D. A. Maria, F. Faião-Flores, D. Michalik, M. C. Marcucci and C. Vogel, "New Antitumoral Agents I: In Vitro Anticancer Activity and in Vivo Acute Toxicity of Synthetic 1,5-Bis(4-hydroxy-3-methoxyphenyl)-1,4-pentadien-3-on e and Derivatives," Bioorganic \& Medicinal Chemistry, Vol. 18, No. 17, 2010, pp. 6275-6281. doi:10.1016/j.bmc.2010.07.026

[49] H. Ligeret, S. Barthélémy, G. B. Doulakas, P. Carrupt, J. Tillement, S. Labidalle and D. Morin, "Fluoride Curcumin Derivatives: New Mitochondrial Uncoupling Agents," FEBS Letters, Vol. 569, No. 1-3, 2004, pp. 37-42. doi:10.1016/j.febslet.2004.05.032

[50] O. Stoetzer, A. Pogrebniak, R. Pelka-Fleischer, M. Hasmann, W. Hiddemann and V. Nuessler, "Modulation of Apoptosis by Mitochondrial Uncouplers: Apoptosis Delaying Features Despite Intrinsic Cytotoxicity," Biochemical Pharmacology, Vol. 63, No. 3, 2002, pp. 471-483. doi:10.1016/S0006-2952(01)00879-6

[51] B. K. Adams, E. M. Ferstl, M. C. Davis, M. Herold, S. Kurtkaya, R. F. Camalier, M. G. Hollingshead, G. Kaur, E. A. Sausville, F. R. Rickles, J. P. Snyder, D. C. Liotta and M. Shoji, "Synthesis and Biological Evaluation of Novel Curcumin Analogs as Anti-Cancer and Anti-Angiogenesis Agents," Bioorganic \& Medicinal Chemistr, Vol. 12, No. 14, 2004, pp. 3871-3883.

doi:10.1016/j.bmc.2004.05.006

[52] A. Sun, M. Shoji , Y. J. Lu, D. C. Liotta and J. P. Snyder, "Synthesis of EF24 Tripeptide Chloromethyl Ketone: A Novel Curcumin-Related Anticancer Drug Delivery System," Journal of Medicinal Chemistry, Vol. 49, No. 11, 2006, pp. 3153-3158. doi:10.1021/jm051141k

[53] A. L. Kasinski, Y. Du, S. L. Thomas, J. Zhao, S. Sun, F. R. Khuri, C. Y. Wang, M. Shoji, A. Sun, J. P. Snyder, D.

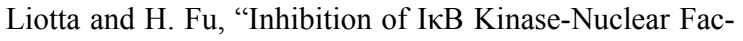
tor- $\kappa \mathrm{B}$ Signaling Pathway by 3,5-Bis(2-flurobenzylidene)piperidin-4-one (EF24), a Novel Monoketone Ana$\log$ of Curcumin," Molecular Pharmacology, Vol. 74, No. 3, 2008, pp. 654-661. doi:10.1124/mol.108.046201

[54] K. M. Youssef, M. A. El-Sherbeny, F. S. El-Shafie, H. A. Farag, O. A. Al-Deeb and S. A. A. Awadalla, "Synthesis of Curcumin Analogues as Potential Antioxidant, Cancer Chemopreventive Agents," Archiv der Pharmazie, Vol. 337, No. 1, 2004, pp. 42-54. doi:10.1002/ardp.200300763

[55] K. M. Youssef and M. A. El-Sherbeny, "Synthesis and Antitumor Activity of Some Curcumin Analogs," Archiv der Pharmazie, Vol. 338, No. 4, 2005, pp. 181-189. doi:10.1002/ardp.200400939

[56] H. Ohori, H. amakoshi, M. Tomizawa, M. Shibuya, Y. Kakudo, A. Takahashi, S. Takahashi, S. Kato, T. Suzuki, C. shioka, Y. Iwabuchi and H. Shibata, "Synthesis and Biological Analysis of New Curcumin Analogues Bearing an Enhanced Potential for the Medicinal Treatment of Cancer," Molecular Cancer Therapeutics, Vol. 5, 2006, pp. 2563-2571. doi:10.1158/1535-7163.MCT-06-0174

[57] H. Shibata, H. Yamakoshi, A. Sato, H. Ohori, Y. Kakudo, C. Kudo, Y. Takahashi, M. Watanabe, H. Takan, C. Ishioka, T. Noda and Y. Iwabuch, "Newly Synthesized Curcumin Analog has Improved Potential to Prevent Colorectal Carcinogenesis in Vivo," Cancer Science, Vol. 100, No. 5, 2009, pp. 956-960. doi:10.1111/j.1349-7006.2009.01127.x

[58] H. Chandru, A. C. Sharada, B. K. Bettadaiah, C. S. A. Kumar, K. S. Rangappa, Sunila and K. Jayashree, "In Vivo Growth Inhibitory and Anti-Angiogenic Effects of Synthetic Novel Dienone Cyclopropoxy Curcumin Analogs on Mouse Ehrlich Ascites Tumor," Bioorganic \& Medicinal Chemistry, Vol. 15, No. 24, 2007, pp. 76967703. doi:10.1016/j.bmc.2007.08.051

[59] J. R. Fuchs, B. Pandit, D. Bhasin, J. P. Etter, N. Regan, D. Abdelhamid, C. Li, J. Lin and P. K. Li, "Structure-Activity Relationship Studies of Curcumin Analogues," Bioorganic \& Medicinal Chemistry Letters, Vol. 19, No. 7, 2009, pp. 2065-2069. doi:10.1016/j.bmcl.2009.01.104

[60] B. Yadav, S. Taurin, R. J. Rosengren, M. Schumacher, M. Diederich, T. J. Somers-Edgar and L. Larsen, "Synthesis and Cytotoxic Potential of Heterocyclic Cyclohexanone Analogues of Curcumin," Bioorganic \& Medicinal Chemistry, Vol. 18, No. 18, 2010, pp. 6701-6707. doi:10.1016/j.bmc.2010.07.063

[61] H. Yamakoshi, H. Ohori, C. Kudo, A. Sato, N. Kanoh, C. Ishioka, H. Shibata and Y. Iwabuchi, "Structure-Activity Relationship of C5-Curcuminoids and Synthesis of Their Molecular Probes Thereof," Bioorganic \& Medicinal Chemistry, Vol. 18, No. 3, 2010, pp. 1083-1092. doi:10.1016/j.bmc.2009.12.045

[62] P. Lagisetty, P. Vilekar, K. Sahoo, S. Anant and V. Awasthi, "CLEFMA-An Anti-Proliferative Curcuminoid from Structure-Activity Relationship Studies on 3,5-Bis(benzylidene)-4-piperidones," Bioorganic \& $\mathrm{Me}$ dicinal Chemistry, Vol. 18, No. 16, 2010, pp. 6109-6120. doi:10.1016/j.bmc.2010.06.055

[63] J. Xiao, Y. Chu, K. Hu, J. Wan, Y. Huang, C. Jiang, G. Liang and X. Li, "Synthesis and Biological Analysis of a New Curcumin Analogue for Enhanced Anti-Tumor Activity in HepG 2 Cells," Oncology Reports, Vol. 23, No. 5, 2010, pp. 1435-1441. doi:10.3892/or 00000781

[64] S. S. Sardjiman, M. S. Reksohadiprodjo, L. Hakim, H. Goot and H. Timmerman, "1,5-Diphenyl-1,4-pentadiene3-ones and Cyclic Analogues as Antioxidative Agents. Synthesis and Structure-Activity Relationship," European Journal of Medicinal Chemistry, Vol. 32, No. 7-8, 1997, pp. 625-630. doi:10.1016/S0223-5234(97)83288-6

[65] K. H. Lee, F. H. A. Aziz, A. Syahida, F. Abas, K. Shaari, D. A. Israf and N. H. Lajis, "Synthesis and Biological Evaluation of Curcumin-Like Diarylpentanoid Analogues 
for Anti-Inflammatory, Antioxidant and Anti-Tyrosinase Activities," European Journal of Medicinal Chemistry, Vol. 44, No. 8, 2009, pp. 3195-3200. doi:10.1016/i.ejmech.2009.03.020

[66] E. Simoni, C. Bergamini, R. Fato, A. Tarozzi, S. Bains, R. Motterlini, A. Cavalli, M. L. Bolognesi, A. Minarini, P. Hrelia, G. Lenaz, M. Rosini and C. Melchiorre, "Polyamine Conjugation of Curcumin Analogues toward the Discovery of Mitochondria-Directed Neuroprotective Agents," Journal of Medicinal Chemistry, Vol. 53, No. 19, 2010, pp. 7264-7268. doi:10.1021/jm100637k

[67] C. Zhao, J. Yang, Y. Wang, D. Liang, X. Yang, X. Li, J. $\mathrm{Wu}, \mathrm{X}$. Wu, S. Yang, X. Li and G. Liang, "Synthesis of Mono-Carbonyl Analogues of Curcumin and Their Effects on Inhibition of Cytokine Release in LPS-Stimulated RAW 264.7 Macrophages," Bioorganic \& Medicinal Chemistry, Vol. 18, No. 7, 2010, pp. 2388-2393. doi:10.1016/j.bmc.2010.03.001
[68] A. M. Katsori, M. Chatzopoulou, K. Dimas, C. Kontogiorgis, A. Patsilinakos, T. Trangas and D. Hadjipavlou-Litina, "Curcumin Analogues as Possible Anti-Proliferative \& Anti-Inflammatory Agents," European Journal of Medicinal Chemistry, Vol. 46, No. 7, 2011, pp. 2722-2735. doi:10.1016/j.ejmech.2011.03.060

[69] G. Liang, S. Yang, L. Jiang, Y. Zhao, L. Shao, J. Xiao, F. Ye, Y. Li and X. Li, "Synthesis and Anti-Bacterial Properties of Mono-carbonyl Analogues of Curcumin," Chemical Pharmaceutical Bulletin, Vol. 56, No. 2, 2008, pp. 162-167. doi:10.1248/cpb.56.162

[70] S. Y. Chen, Y. Chen, Y. P. Li, S. H. Chen, J. H. Tan, T. M. Ou, L. Q. Gu and Z. S. Huang, "Design, Synthesis, and Biological Evaluation of Curcumin Analogues as Multifunctional Agents for the Treatment of Alzheimer's disease," Bioorganic \& Medicinal Chemistry, Vol. 19, No. 18, 2011, pp. 5596-5604. doi:10.1016/j.bmc.2011.07.033 